\title{
Effect of Surfactant on Package Substrate in Chemical Mechanical Planarization
}

\author{
Soocheon Jang ${ }^{1,2}$, Haedo Jeong ${ }^{1, \#,}$, Minjong Yuh', and Jaehong Park \\ 1 School of Mechanical Engineering, Pusan National University, 2, Busandaehak-ro, 63beon-gil, Geumjeong-gu, Busan, South Korea, 609-836 \\ 2 G\&P Technology, \#304 MEMSNANO Center, Pusan National University, 2, Busandaehak-ro, 63beon-gil, Geumjeong-gu, Busan, South Korea, 609-836 \\ 3 Nitta Haas Inc., 3-17-1, Kannabidai, Kyotanabe-shi, Kyoto, Japan, 610-0333 \\ \# Corresponding Author / E-mail: hdjeong@pusan.ac.kr, TEL: +82-51-510-3210, FAX: +82-51-518-8442
}

KEYWORDS: Chemical mechanical planarization(CMP), Dishing, Erosion, Package substrate, Surfactant

\begin{abstract}
The demand for pattern miniaturization on package substrates has been steadily increasing. One technical innovation for the package substrate manufacturing process was chemical mechanical planarization (CMP). In conventional wiring, it was possible to remove extraneous copper through only the etching process. However, etching defects occur with narrower line widths. As the package substrate has a $\mathrm{Cu}$ hybrid structure through $\mathrm{Cu}$ plating and copper clad laminate (CCL) removal, it is necessary to apply the CMP to remove excess copper. However, defects are generated by the CMP process due to mechanical and chemical effects from the slurry. This study investigated the surfactant effect on $\mathrm{Cu}$ dishing and erosion in patterns with approximately 10/10 um line width and spacing. The conventional Cu slurry without a surfactant had severe erosion $(0.58 \mu \mathrm{m})$ in $\mathrm{Cu}$ patterns of $4 / 6 \mu \mathrm{m}$ and deep dishing $(4.2 \mu \mathrm{m})$ in Cu patterns of 14/16 $\mu \mathrm{m}$. However, the experimental results showed that the friction force during CMP decreased, with smaller dishing and erosion as surfactant concentration increased. Finally, globally planarized Cu patterns were realized with an erosion range of $0.22 \mu \mathrm{m}$ to $0.35 \mu \mathrm{m}$ and a dishing range of $0.37 \mu \mathrm{m}$ to $0.69 \mu \mathrm{m}$ with $3 \mathrm{wt} \%$ surfactant.
\end{abstract}

\section{Introduction}

The demand for electronic package technology that can mount semiconductor elements more effectively is increasing due to the demand for increasingly lighter, smaller, multi-functional mobile devices and electronic goods resulting from recent advancements in the information and communication industries. ${ }^{1-3}$ Previously, signals were transmitted through wire bonding used to connect the chip to the substrate. However, this method faces a number of problems such as decreased signal transmission speed, decreased high frequency wave characteristics, and an input/output (I/O) pad increase resulting from high resistance as the overall wiring length increased. ${ }^{4}$ Recently, efforts have been made to address the weaknesses induced by wire bonding through improved high density integration, high speed signal transmission, electrical conductivity, and heat conduction characteristics through the copper plating process and by vias and wiring on the package substrate. ${ }^{5}$

The fabrication of narrower and shallower wires on the package substrate has been limited due to the application of a sustainable etching process that has been used previously. In order to realize fine patterns below $10 \mu \mathrm{m}$ on the package substrate, the chemical mechanical planarization (CMP) process is considered one of the best planarization technologies for the semiconductor process. ${ }^{6}$

The package substrate has a copper hybrid laminating structure, fabricated through a repetitive plating and removal process on the copper clad laminate (CCL). Recently, CMP technology was introduced to remove extraneous copper from the substrate surface. ${ }^{4,7}$ The package substrate was made by laser ablation of a dielectric material comprised of epoxy resin and glass fiber, which then goes through a $\mathrm{Cu}$ plating and CMP process. The typical types of defect that can be generated during the CMP process as it removes the plating film are shown in Fig. 1. The main defects are a $\mathrm{Cu}$ dishing resulting from the differences in material removal rates, and dielectric erosion from the dynamic relationship of the process elements with an excessive removal phenomenon. These defects can affect the processing or structural characteristics, leading to wiring short circuits during the laminating process. ${ }^{8}$

Accordingly, excessive $\mathrm{Cu}$ dishing and dielectric erosion must be minimized in order to realize a finer line width. ${ }^{8-12}$ One solution to minimize these defects is the use of a surfactant, described herein.

Surfactants are generally used to reduce surface tension by forming 

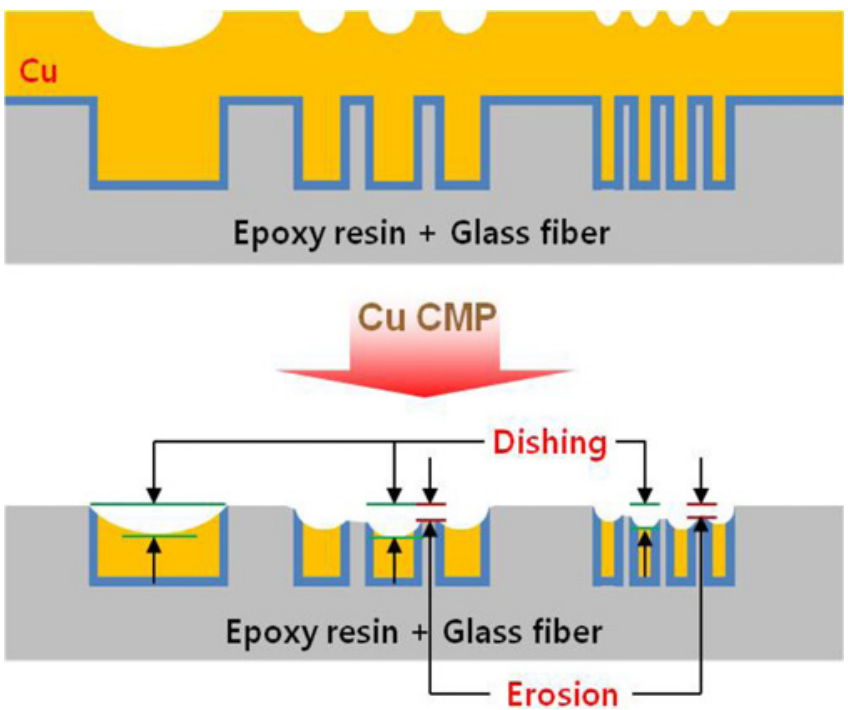

Fig. 1 Dielectric erosion and $\mathrm{Cu}$ dishing after $\mathrm{Cu} \mathrm{CMP}$
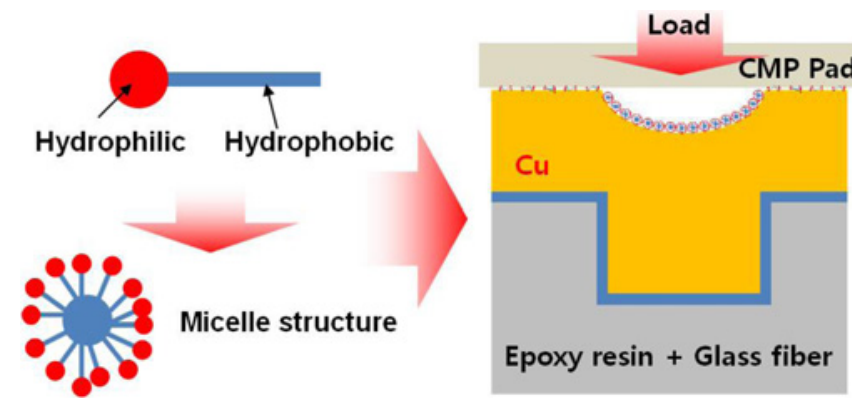

Fig. 2 Effects of micelle structure (left) on the CMP process (right)

micelle structures around the hydrophobic or hydrophilic regions of a molecule. The surfactant possesses both a hydrophilic head group and hydrophobic tail. Fig. 2 shows the micelle structure formed by the hydrophobic tails sticking together with the hydrophilic head groups facing outwards. On the projecting $\mathrm{Cu}$ areas contacting the CMP pad, the surfactant's micelle structure collapses, allowing removal of the $\mathrm{Cu}$ by the mechanical action of the abrasive particles with the pad during the CMP process. However, the recessed $\mathrm{Cu}$ regions are protected from chemical reaction by the micelle structures. The surfactant was also added onto the slurry to verify how effectively the projecting copper on the package substrate was removed during the CMP process. In order to optimize the amount of surfactant, the resultant $\mathrm{Cu}$ dishing and dielectric erosion were observed, which could explain the planarity level achieved during the CMP process. ${ }^{13-15}$

\section{Experimental Conditions}

The CMP has a synergistic effect of chemical dissolution (or passivation) with mechanical abrasion by the slurry between the pad and the package substrate. A kinematic motion results from the relative velocities of the head and platen rotation. ${ }^{16}$ The quantity of $\mathrm{Cu}$ dishing and dielectric erosion were measured after the CMP using a slurry containing $1 w t . \%$ ammonia, $3 w t . \%$ colloidal silica $(70 \mathrm{~nm}), 5 w t . \%$ organic acid, and $2.3 w t . \%$ hydrogen peroxide relative to deionized (DI) water.

In general, the amount of $\mathrm{Cu}$ dishing decreases as the line width
Table 1 The slurry additive compositions

\begin{tabular}{ccc}
\hline Additive & Concentration $(w t \%)$ \\
\hline Ammonia & $1 w t \%$ \\
\hline Colloidal silica(70 nm) & $3 w t \%$ \\
\hline Organic acid & $5 w t \%$ \\
\hline Hydrogen peroxide & & $2.3 w t \%$ \\
\cline { 2 - 3 } Surfactant & $\# 1$ & Non-additive \\
\cline { 2 - 3 } (linear alkylbenzene & $\# 2$ & $0.1 w t \%$ \\
\cline { 2 - 3 } sulphonate) & $\# 3$ & $1 w t \%$ \\
\cline { 2 - 3 } & $\# 4$ & $2 w t \%$ \\
\cline { 2 - 3 } & $\# 5$ & $3 w t \%$ \\
\hline
\end{tabular}

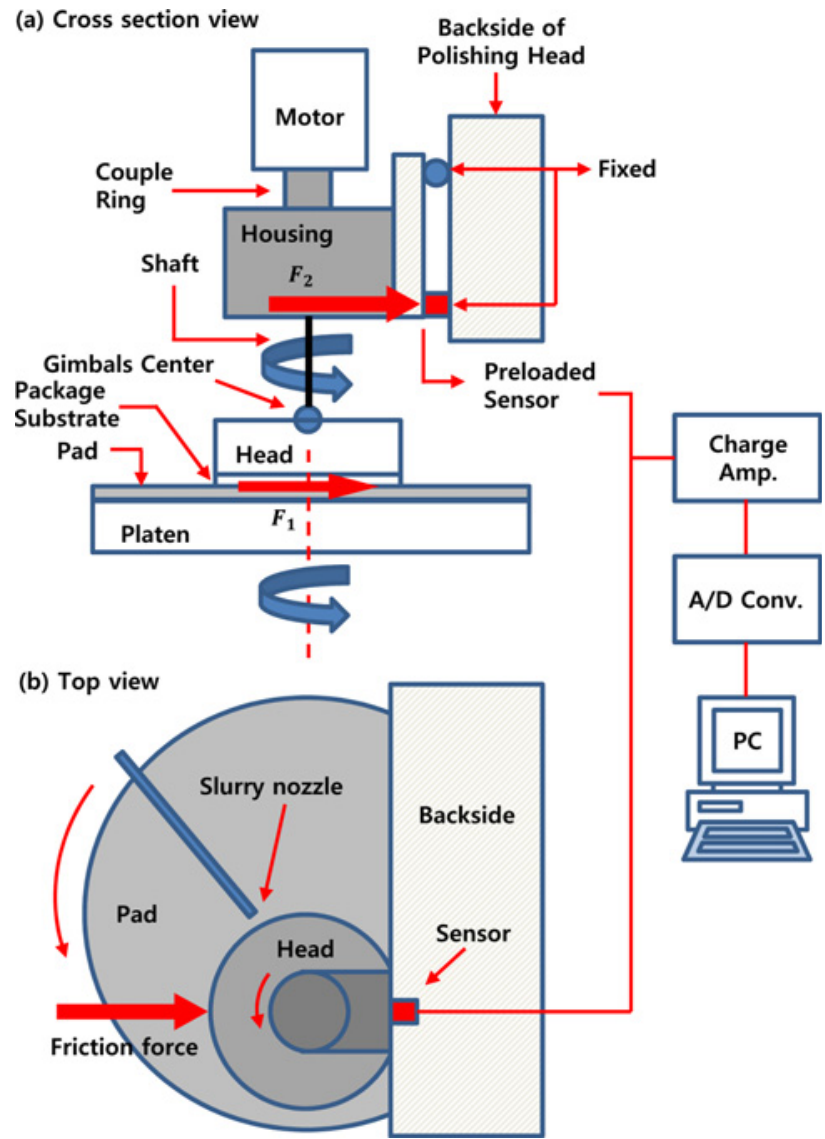

Fig. 3 Schematic of the friction monitoring system showing (a) the cross section view, and (b) the top view

decreases in semiconductor device nanopatterns while the amount of dielectric erosion increases. However, excessive dielectric erosion increases significantly as the nanopattern approached fine line widths, while the amount of $\mathrm{Cu}$ dishing also increased. To minimize these defects, a traditional anionic surfactant, linear alkylbenzene sulfonate (LAS), was added into the slurry for polishing. The different amounts of surfactant added are shown in Table 1. A pre-CMP package substrate was attached with an adhesive film. The package substrate was comprised of copper patterns with line and spacing widths of $4 / 6 \mu \mathrm{m}, 6 / 8 \mu \mathrm{m}, 9 / 11 \mu \mathrm{m}$, and $14 /$ $16 \mu \mathrm{m}$. To indirectly verify that there has been a change in surface tension and adhesion due to the surfactant, a friction monitoring system was used to measure the frictional force generated at the contact point by the pressure and velocity of the head and platen during the CMP.

Fig. 3 shows a schematic of the monitoring system installed in the CMP equipment. The frictional force is vertical to the head's rotational 
Table 2 Experimental conditions

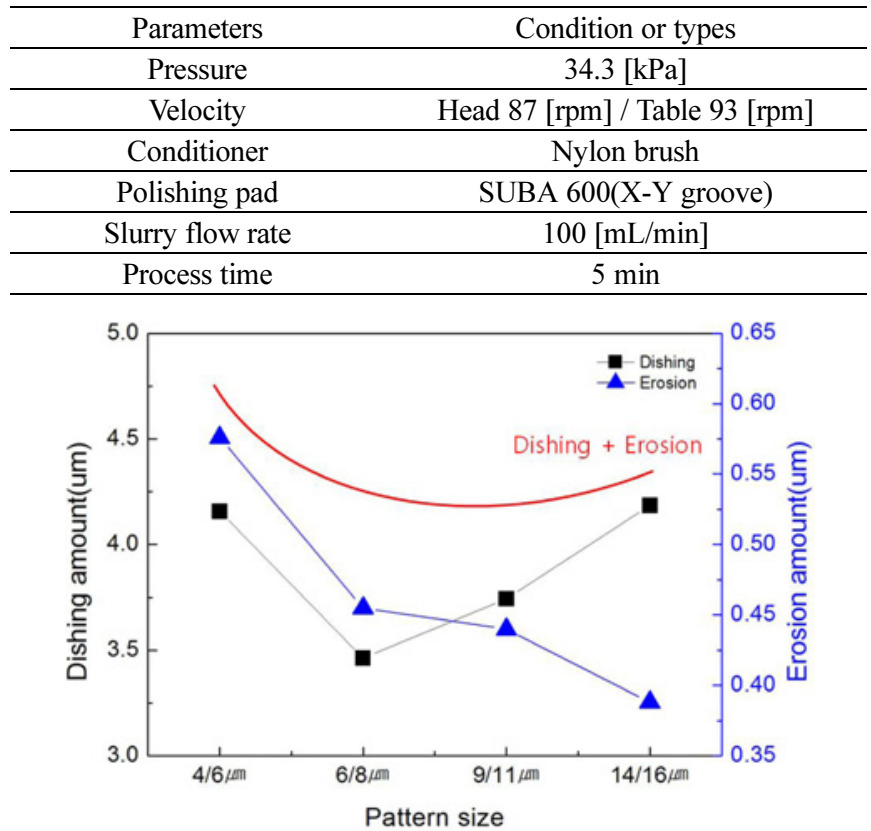

Fig. 4 Amount of dielectric erosion and $\mathrm{Cu}$ dishing without surfactant in the $\mathrm{Cu} \mathrm{CMP}$

axis without any movement generated in real-time. The frictional force signal is converted by the amplifier and analog to digital (A/D) converter, and then manifested through a sensor.

Fig. 3 shows a schematic of the monitoring system installed in the CMP equipment. The frictional force is vertical to the head's rotational axis without any movement generated in real-time. The frictional force signal is converted by the amplifier and analog to digital (A/D) converter, and then manifested through a sensor correction. ${ }^{11}$ In order to verify the frictional force change relative to the surfactant amount added in the slurry, a CCL without any micropattern was used. The experimental conditions are shown in Table 2. The package substrate was pressurized under $34.3 \mathrm{kPa}$ with an $87 \mathrm{rpm}$ speed on the polyurethane non-woven pad with X-Y grooves for $5 \mathrm{~min}$. The slurry was supplied from the center of the pad with a $100 \mathrm{~mL} / \mathrm{min}$ flow rate. Next, the quantity of $\mathrm{Cu}$ dishing and dielectric erosion on the package substrate surface after CMP was observed with a confocal microscope to analyze the effect of the surfactant.

\section{Results and Discussion}

Fig. 4 is a graph displaying the results of no surfactant in the slurry. It can be seen that dishing from 3.46 to $4.19 \mu \mathrm{m}$ and erosion from 0.39 to $0.58 \mu \mathrm{m}$ occurred depending on the pattern size. Excessive dielectric erosion was shown in Fig. 4 as the insulator wore away significantly as it approached fine line widths, and the amount of $\mathrm{Cu}$ dishing increased as well.

\subsection{Friction}

To verify the effect of surfactant on the friction force during CMP, a friction force sensor was attached to the testing equipment and its changes were monitored. Fig. 5 shows a change of the average

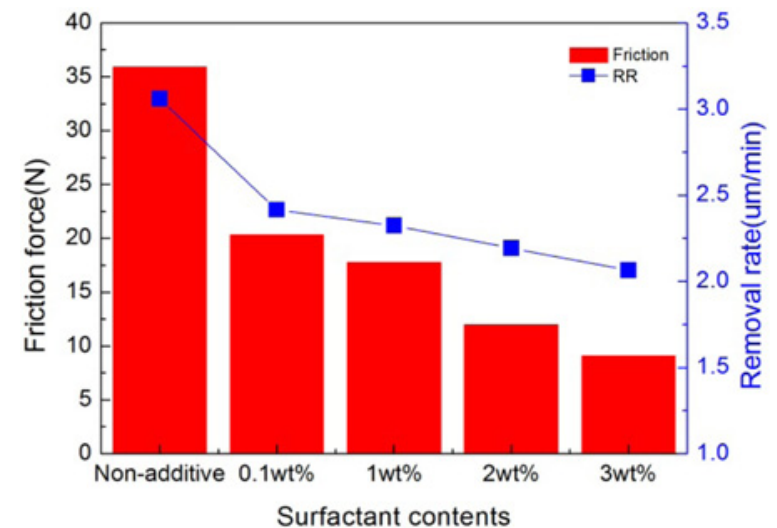

Fig. 5 The friction force and removal rate according to surfactant concentration during CMP

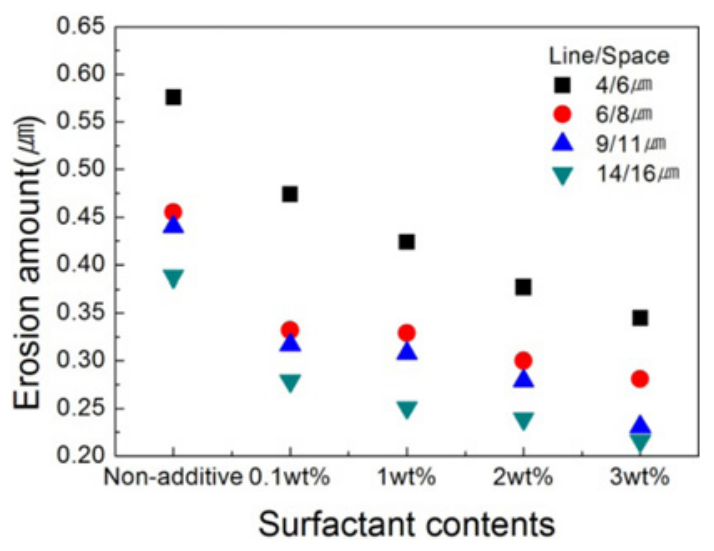

Fig. 6 Dielectric erosion amount according to surfactant contents

frictional force, displaying a constant decrease as the amount of surfactant increased. This indicates that the surfactant alleviated the frictional force generated at the interface between the copper pattern and the pad during CMP.

\subsection{Erosion}

Overpolishing is necessary to completely remove $\mathrm{Cu}$ residue from the entire surface; however, this makes the dielectric erosion worse. Fig. 6 is a graph presenting the amount of dielectric erosion after CMP relative to surfactant concentration. The dielectric erosion steadily reduced relative to the amount of surfactant. This phenomenon has a good correlation with the frictional force changes during CMP with added surfactant.

Moreover, the amount of dielectric erosion increased consistently with decreasing line widths in Fig. 6 and Fig. 7. The dielectric erosion was worse for narrower spacing compared to wider spacing. Namely, the amount of dielectric erosion increased as the line width became narrower, which is why the narrow patterned insulator could not withstand the polishing pressure, rapidly eroding away.

\subsection{Dishing}

Fig. 8 shows the amount of $\mathrm{Cu}$ dishing after $\mathrm{CMP}$ with varied amounts of surfactant. A significant amount of $\mathrm{Cu}$ dishing (3.5-4.2 $\mu \mathrm{m})$ took place without the presence of surfactant. When $0.1 \mathrm{wt} \%$ surfactant was added, the $\mathrm{Cu}$ dishing reduced significantly compared to that without surfactant. The amount of $\mathrm{Cu}$ dishing gradually reduced to 


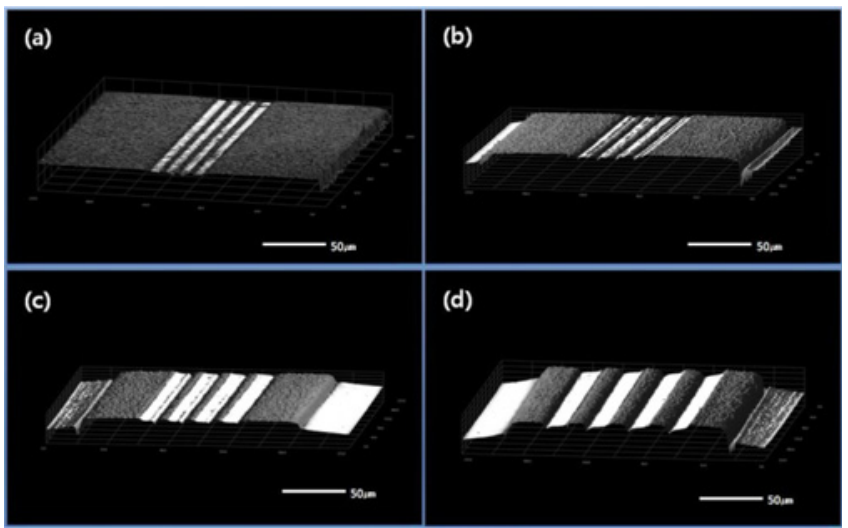

Fig. 7 Confocal microscope images of the $\mathrm{Cu}$ patterns at (a) $4 / 6 \mu \mathrm{m}$, (b) $6 / 8 \mu \mathrm{m}$, (c) $9 / 11 \mu \mathrm{m}$, and (d) $14 / 16 \mu \mathrm{m}$ after CMP with $3 w t . \%$ surfactant

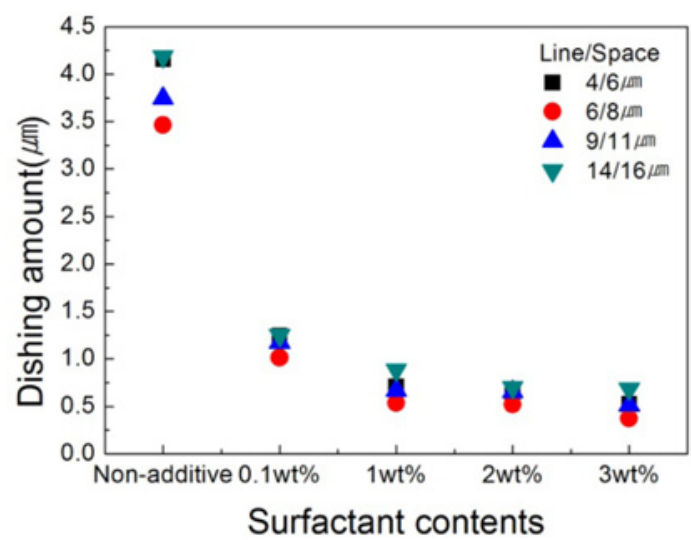

Fig. $8 \mathrm{Cu}$ dishing amount according to surfactant contents

approximately $0.5 \mu \mathrm{m}$ when the amount of surfactant was increased to $3 w t . \%$. Finally, it was confirmed that the $\mathrm{Cu}$ dishing was primarily dependent on the amount of surfactant, regardless of changes in the line width. Moreover, the amount of $\mathrm{Cu}$ dishing also depended on the amount of dielectric erosion.

\section{Conclusions}

This study examined the post-CMP surface defects in copper patterns with line and spacing widths of $4 / 6 \mu \mathrm{m}, 6 / 8 \mu \mathrm{m}, 9 / 11 \mu \mathrm{m}$, and $14 / 16 \mu \mathrm{m}$ on the package substrate surface. In particular, it was possible to verify that the surfactant in the CMP slurry directly affected the amount of surface defects. Without surfactant, a relatively high frictional force was generated, leading to material overpolishing and generating process defects such as $\mathrm{Cu}$ dishing and dielectric erosion. On the other hand, with the inclusion of a surfactant, a relatively low frictional force was achieved, significantly reducing the $\mathrm{Cu}$ dishing and dielectric erosion amounts.

\section{ACKNOWLEDGEMENT}

This research was financially supported by the Small and Medium Business Administration(SMBA) through R\&D program for small and medium-sized enterprises(S2089351).

\section{REFERENCES}

1. Hung, K., Chan, Y., Ong, H., Tu, P., and Tang, C., "Effect of Pinhole $\mathrm{Au} / \mathrm{Ni} / \mathrm{Cu}$ Substrate on Self-Alignment of Advanced Packages," Materials Science and Engineering: B, Vol. 76, No. 2, pp. 87-94, 2000.

2. Lee, H.-J., Ji, C.-W., Woo, S.-M., Choi, M.-H., Hwang, Y.-H., et al., "Formation of Copper Seed Layers and Copper Via Filling with Various Additives," The Materials Research Society of Korea, Vol. 22, No. 7, pp. 335-341, 2012.

3. Kobayashi, T., Kawasaki, J., Mihara, K., and Honma, H., "ViaFilling using Electroplating for Build-Up PCBs," Electrochimica Acta, Vol. 47, No. 1, pp. 85-89, 2001.

4. Siau, S., Vervaet, A., Degrendele, L., Baets, J. D., and Calster, A.V., "Qualitative Electroless Ni/Au Plating Considerations for the Solder Mask on Top of Sequential Build-Up Layers," Applied Surface Science, Vol. 252, No. 8, pp. 2717-2740, 2006.

5. Rossetti, A., Codeluppi, R., Zagnoni, M., Talamelli, A., and M. Tartagni, "A PCB-Embedded Pressure Sensor for Wire Sail Monitoring,” Procedia Engineering, Vol. 5, pp. 315-318, 2010.

6. Lee, H., Dornfeld, D. A., and Jeong, H., "Mathematical ModelBased Evaluation Methodology for Environmental Burden of Chemical Mechanical Planarization Process,” Int. J. Precis. Eng. Manuf.-Green Tech., Vol. 1, No. 1, pp. 11-15, 2014.

7. Ra, S., Lee, C., Cho, J., Lee, S., Lee, J., et al., "Micro Via and Line Patterning for PCB Using Imprint Technique," Current Applied Physics, Vol. 8, No. 6, pp. 675-678, 2008.

8. Noh, K., Saka, N., and Chun, J.-H., "Effect of Slurry Selectivity on Dielectric Erosion and Copper Dishing in Copper ChemicalMechanical Polishing," CIRP Annals-Manufacturing Technology, Vol. 53, No. 1, pp. 463-466, 2004.

9. Saka, N., Lai, J.-Y., Chun, J.-H., and Sun, N. P., "Mechanisms of the Chemical Mechanical Polishing (CMP) Process in Integrated Circuit Fabrication," CIRP Annals-Manufacturing Technology, Vol. 50, No. 1, pp. 233-238, 2001.

10. Byrne, G., Mullany, B., and Young, P., "The Effect of Pad Wear on the Chemical Mechanical Polishing of Silicon Wafers," CIRP Annals-Manufacturing Technology, Vol. 48, No. 1, pp. 143-146, 1999.

11. Lee, H., Park, B., and Jeong, H., "Influence of Slurry Components on Uniformity in Copper Chemical Mechanical Planarization," Microelectronic Engineering, Vol. 85, No. 4, pp. 689-696, 2008.

12. Lee, H., Joo, S., and Jeong, H., "Mechanical Effect of Colloidal Silica in Copper Chemical Mechanical Planarization," Journal of Materials Processing Technology, Vol. 209, No. 20, pp. 6134-6139, 2009.

13. Longo, M. A. and Combes, D., "Influence of Surface Hydrophilic/ Hydrophobic Balance on Enzyme Properties," Journal of Biotechnology, Vol. 58, No. 1, pp. 21-32, 1997. 
14. Neirynck, J. M., Yang, G.-R., Murarka, S. P., and Gutmann, R. J., "The Addition of Surfactant to Slurry for Polymer CMP: Effects on Polymer Surface, Removal Rate and Underlying Cu,” Thin Solid Films, Vol. 290, pp. 447-452, 1996.

15. Bernard, P., Kapsa, P., Coudé, T., and Abry, J.-C., "Influence of Surfactant and Salts on Chemical Mechanical Planarisation of Copper," Wear, Vol. 259, No. 7, pp. 1367-1371, 2005.

16. Kim, H. and Jeong, H., "Effect of Process Conditions on Uniformity of Velocity and Wear Distance of Pad and Wafer during Chemical Mechanical Planarization,” Journal of Electronic Materials, Vol. 33, No. 1, pp. 53-60, 2004. 